\title{
Strength of the Effective Coulomb Interaction at Metal and Insulator Surfaces
}

\author{
Ersoy Şaşığlu, ${ }^{*}$ Christoph Friedrich, and Stefan Blügel \\ Peter Grünberg Institut and Institute for Advanced Simulation, Forschungszentrum Jülich and JARA, 52425 Jülich, Germany
}

(Received 29 May 2012; published 1 October 2012)

\begin{abstract}
The effective on-site Coulomb interaction (Hubbard $U$ ) between localized electrons at crystal surfaces is expected to be enhanced due to the reduced coordination number and reduced subsequent screening. By means of first principles calculations employing the constrained random-phase approximation we show that this is indeed the case for simple metals and insulators but not necessarily for transition metals and insulators that exhibit pronounced surface states. In the latter case, the screening contribution from surface states as well as the influence of the band narrowing increases the electron polarization to such an extent as to overcompensate the decrease resulting from the reduced effective screening volume. The Hubbard $U$ parameter is thus substantially reduced in some cases, e.g., by around $30 \%$ for the (100) surface of bcc Cr.

DOI: 10.1103/PhysRevLett.109.146401

PACS numbers: 71.15. $-\mathrm{m}, 71.10 . \mathrm{Fd}, 71.28 .+\mathrm{d}$
\end{abstract}

The effective on-site Coulomb interaction (Hubbard $U$ ) between localized electrons at surfaces of solids is expected to be enhanced since the effective screening volume of the surface is reduced with respect to the bulk. As a consequence, the electron polarization decreases at the surface, which reduces the effect of screening and gives rise to a larger $U$ value. These arguments are underscored by interpolating between the Hubbard $U$ values of an isolated atom and an atom in a bulk solid, the former being 3 to 5 times larger than the latter [1]. Neither experimental nor theoretical works have been reported so far that would address the strength of the surface $U$ parameter explicitly. However, a large number of phenomena observed in solids indicates an enhancement of the $U$ at surfaces. For instance, the metal-insulator transition at the surface of correlated materials [2], the appearance of magnetism at the surface of paramagnetic transition metals [3], and the enhanced exchange splitting at the surface of $3 d$ ferromagnets [4] have been attributed to an increase of the correlation strength, which is defined by the ratio $U / W$, where $W$ is the bandwidth. In the theoretical description of the surfaces, the $U$ is usually assumed to be unchanged [5-7] so that the enhancement of correlation at the surface $(S)$ with respect to the bulk $(B)$ is provided by the effective band narrowing, i.e., $W_{S}<W_{B}$. In principle, depending on the relative values of the surface $U$ and the bandwidth $W$, the correlation strength can further increase or decrease even below the bulk value. However, the latter case is considered to be unlikely because it is believed that $U$ always increases at surfaces. In this Letter, we show by means of first-principles calculations that contrary to this conventional wisdom, this is not always the case. It decreases at many transition-metal (TM) surfaces and insulator surfaces with pronounced surface states, as a result of additional screening channels that open up due to surfacerelated changes in the electronic structure.

Recently, the calculation of the Hubbard $U$ parameter in solids from first principles has been addressed by several authors [8-15]. A number of different approaches have been proposed and applied to the bulk phase of various classes of materials. However, the effective Coulomb interaction at a surface has been considered only within a model Hamiltonian framework [16], so far. Reining and Del Sole [17] performed model calculations to account for the contribution of the surface states to the static electronic screening at the $\mathrm{Si}(111)$ surface. The authors showed that the surface states give rise to a substantial enhancement of electron screening at the surface, reducing the correlation strength.

The aim of this Letter is to determine the strength of the on-site effective Coulomb interaction between localized electrons at metal and insulator surfaces from first principles. To calculate the Hubbard $U$ parameter we employ the constrained random-phase approximation (cRPA) [11] within the full-potential linearized augmented-plane-wave method using maximally localized Wannier functions (MLWFs) [12,18]. Our calculations show that the Hubbard $U$ parameter is enhanced at simple metal $(\mathrm{Na}, \mathrm{Al})$ and most insulator $\left(\mathrm{SrTiO}_{3}, \mathrm{NaCl}\right)$ surfaces with respect to bulk as expected. However, the situation is different for TMs and insulators with pronounced surface states. For TMs, both the interplay of the surface states and the effective band narrowing can give rise to a substantial reduction of the Hubbard $U$, while for insulators only the surface states are responsible for the reduction of $U$. For the (100) surface of bcc $\mathrm{Cr}$ we obtain a $30 \%$ reduction of the static $U$. Moreover, the frequency dependence $U(\omega)$ is markedly different from that of the bulk. For bcc $\mathrm{Cr}$ we find that, starting from $\omega=0$, the effective Coulomb interaction $U(\omega)$ increases monotonically with frequency, and at about $2 \mathrm{eV}$ it exceeds the bulk value, which remains basically constant in this interval.

We model the metal and insulator surfaces with slabs of 11 atomic layers. Such slabs form a superlattice with $20 \AA$ of vacuum separating them, with each slab possessing two (100) or (110) symmetric surfaces. To discuss the chemical 
trends, additional modifications such as surface reconstruction and surface relaxation are at first not taken into account. We consider the $3 d \mathrm{TMs}$ in their respective ground-state crystal structures except $\mathrm{Sc}, \mathrm{Ti}$, and $\mathrm{Co}$ $(\mathrm{Mn})$, which are treated in the fcc (bcc) structure. The ground-state calculations are carried out using the fullpotential linearized augmented-plane-wave method as implemented in the FLEUR code [19] with the generalized gradient approximation (GGA) exchange-correlation potential as parameterized by Perdew et al. [20]. The MLWFs are constructed with the WANNIER 90 code $[21,22]$. The effective Coulomb potential is calculated within the recently developed cRPA method [11] implemented in the SPEX code [23] (for further technical details see Refs. [12,24]).

The cRPA approach offers an efficient way to calculate the effective Coulomb interaction $U$ and allows us to determine individual Coulomb matrix elements, e.g., on site, off site, intraorbital, interorbital, and exchange as well as their frequency dependence. The basic idea behind the cRPA [11] is to define an effective interaction $U$ between the localized electrons by restricting the screening processes to those that are not explicitly treated in the effective model Hamiltonian. To this end, the full RPA polarization matrix $P$ is divided into $P=P_{l}+P_{r}$, where $P_{l}$ includes only transitions between the localized states, for which the Hubbard $U$ is to be calculated, and $P_{r}$ is the remainder. Thus, the localized states are largely eliminated, and the screening is dominated by itinerant $s$ and $p$ states, which are well described by LDA and GGA. The cRPA is, therefore,- - even with these standarad $x c$ potentialsconsidered a reliable approach to calculate the Hubbard $U$ parameter and its frequency dependence [25,26]. Then, the frequency-dependent effective Coulomb interaction is given schematically by the matrix equation $U(\omega)=$ $\left[1-v P_{r}(\omega)\right]^{-1} v$, where $v$ is the bare Coulomb interaction. The static limit of the average diagonal matrix element of $U(\omega \rightarrow 0)$ represented in a local basis can then be regarded as the Hubbard $U$ parameter. The matrix elements of the effective Coulomb potential $U$ in the MLWF basis are given by $U_{\mathbf{R} n_{1} n_{3} ; n_{4} n_{2}}(\omega)=\int w_{n_{1} \mathbf{R}}^{*}(\mathbf{r}) \times$ $w_{n_{3} \mathbf{R}}(\mathbf{r}) U\left(\mathbf{r}, \mathbf{r}^{\prime} ; \omega\right) w_{n_{4} \mathbf{R}}^{*}\left(\mathbf{r}^{\prime}\right) w_{n_{2} \mathbf{R}}\left(\mathbf{r}^{\prime}\right) d^{3} r d^{3} r^{\prime}$, where $w_{n \mathbf{R}}(\mathbf{r})$ is the MLWF at site $\mathbf{R}$ with orbital index $n$ and $U\left(\mathbf{r}, \mathbf{r}^{\prime} ; \omega\right)$ is calculated within the cRPA. We define the average on-site diagonal (direct intra-orbital) and off-diagonal (exchange inter-orbital) matrix elements of the effective Coulomb potential as $U=L^{-1} \sum_{n} U_{\mathbf{R} n n ; n n}$ and $J=$ $[L(L-1)]^{-1} \sum_{m, n(m \neq n)} U_{\mathbf{R} m n ; n m}$, where $L$ is the number of localized orbitals, i.e., one, three, and five for $s, p$, and $d$ states, respectively.

In Fig. 1 we present the calculated layer dependence of the Hubbard $U$ for three different systems: (a) for the $3 p$ states of the simple metal fcc $\mathrm{Al}$, (b) the $3 d$ states of the transition metal bcc $\mathrm{Cr}$, and (c) the Cl-3p states of the insulator $\mathrm{NaCl}$ in the rock-salt (RS) structure. As seen in
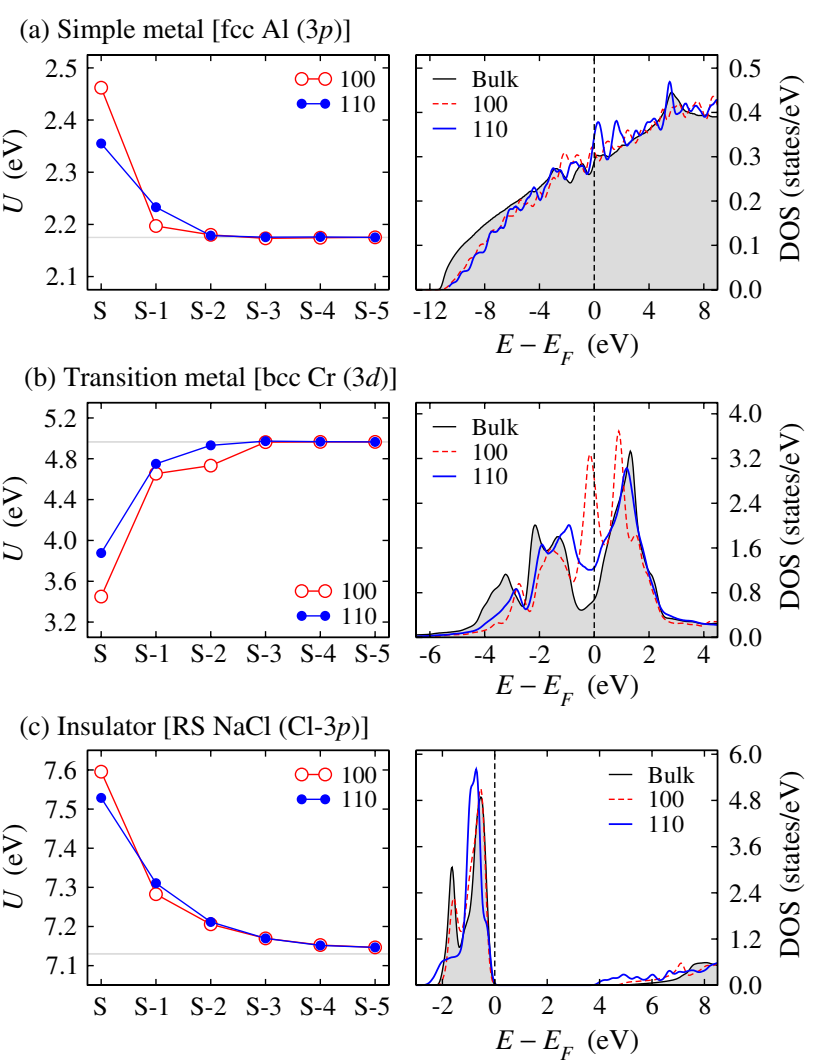

FIG. 1 (color online). (a) Left panel: Layer dependence of the Hubbard $U$ parameter for fcc Al. The $S$ denotes the surface layer in the slab model. Right panel: Total density of states (DOS) for the (100) and (110) surface of fcc Al. For comparison, the bulk DOS (shaded area) is included; (b) and (c) the same for bcc $\mathrm{Cr}$ and rock-salt $\mathrm{NaCl}$.

cases (a) and (c), the Hubbard $U$ increases from the middle layer, where it is close to the bulk value, to the surface layer as expected. However, we find an unexpected behavior in the case of bcc $\mathrm{Cr}$, where $U$ is substantially reduced at the surface. This reduction is $30 \%$ for the open (100) and $20 \%$ for the (110) surface. For simplicity-the magnetism of bcc $\mathrm{Cr}$ is quite complicated-we only consider the nonmagnetic state here. Furthermore, the layer dependence of $U$ is quite different in metals and insulators. Because of the short screening length in metals, the Hubbard $U$ quickly assumes the bulk value in the former, as we go from the surface toward the middle of the slab, while in the latter the layer-by-layer convergence to the bulk value is much slower. On the other hand, the surface $J$ values, which are listed in Table I for various materials, only differ slightly from the corresponding bulk values.

We find that the surface electronic polarization and, as a consequence, the Hubbard $U$ parameter are determined by two competing effects. (i) The so-called dimensionality effect, which is due to the reduced coordination number and hence the decrease of the effective screening volume at the surface region. From the point of view of classical electrostatics, this effect reduces the electronic polarization 
TABLE I. Bulk and surface Hubbard $U$ and $J$ values for simple metals and insulators. The corresponding orbitals for which the $U$ and $J$ are calculated are given in parentheses.

\begin{tabular}{lcccccc}
\hline \hline & Bulk & \multicolumn{3}{c}{ 100 Surface } & \multicolumn{3}{c}{ 110 Surface } \\
\hline & $U_{B}$ & $J_{B}$ & $U_{S}$ & $J_{S}$ & $U_{S}$ & $J_{S}$ \\
\hline $\mathrm{Na}[3 s]$ & 1.39 & & 1.50 & & 1.47 & \\
$\mathrm{Al}[3 p]$ & 2.18 & 0.36 & 2.46 & 0.39 & 2.36 & 0.38 \\
$\mathrm{MgO}[\mathrm{O}-2 p]$ & 7.10 & 0.63 & 7.23 & 0.62 & 6.38 & 0.59 \\
$\mathrm{NaCl}[\mathrm{Cl}-3 p]$ & 7.13 & 0.55 & 7.60 & 0.54 & 7.53 & 0.55 \\
$\mathrm{SrTiO}_{3}[\mathrm{Ti}-3 d]$ & 3.34 & 0.37 & 3.62 & 0.39 & & \\
$\mathrm{SrTiO}_{3}[\mathrm{O}-2 p]$ & 4.42 & 0.56 & 4.79 & 0.55 & & \\
\hline \hline
\end{tabular}

at the surface leading to larger $U$ values. (ii) Electronic structure effects, i.e., the appearance of surface states and the effective band narrowing. This second effect gives rise to an enhancement of the electronic polarization and, hence, to a decrease of $U$. Depending on the strength of the two competing effects, the effective Coulomb interaction at the surface can be enhanced as well as reduced with respect to the bulk value. Qualitative information on the influence of the surface electronic structure on the Hubbard $U$ parameter, leading to the second effect, can be deduced from the polarization function, which is given by

$$
\begin{aligned}
P\left(\mathbf{r}, \mathbf{r}^{\prime} ; \omega\right)= & 2 \sum_{m}^{\text {occ unocc }} \sum_{m^{\prime}} \psi_{m}(\mathbf{r}) \psi_{m^{\prime}}^{*}(\mathbf{r}) \psi_{m}^{*}\left(\mathbf{r}^{\prime}\right) \psi_{m^{\prime}}\left(\mathbf{r}^{\prime}\right) \\
& \times\left[\frac{1}{\omega-\Delta_{m m^{\prime}}+i \delta}-\frac{1}{\omega+\Delta_{m m^{\prime}}-i \delta}\right],
\end{aligned}
$$

where $\psi_{m}(\mathbf{r})$ and $\epsilon_{m}$ are Kohn-Sham eigenfunctions and eigenvalues, respectively, $\delta$ is a positive infinitesimal, and $\Delta_{m m^{\prime}}=\epsilon_{m^{\prime}}-\epsilon_{m}$. For zero frequency $(\omega=0)$ the main contribution to the polarization function comes from the states around the Fermi energy. The smaller the energy difference between occupied and unoccupied states the larger the contribution. The effective band narrowing in TMs tends to reduce $\Delta_{m m^{\prime}}^{S}$ with respect to $\Delta_{m m^{\prime}}^{B}$, which has the effect of increasing the polarization. Additionally, the presence of surface states close to the Fermi level at the TM bcc (100) surfaces makes $\Delta_{m m^{\prime}}^{S}$ effectively smaller resulting in a more efficient electronic polarization and, as a result, in substantially reduced $U$ and $\tilde{U}$ values as shown in Fig. 2 for the $3 d$ series, where $\tilde{U}$ stands for the fully screened Coulomb interaction within the RPA. As seen in Fig. 1, the density of states (DOS) of the (100) surface of bcc $\mathrm{Cr}$ exhibits a pronounced peak that is caused by a surface state just below the Fermi level, which is also found in previous first-principles calculations [27]. This surface state contributes substantially to the polarization function and is mainly responsible for the $10 \%$ stronger reduction of the $U$ value compared to the corresponding value for the (110) surface, where such a peak in the DOS is missing. On the other hand, in simple metals the surface electronic structure turns out to be very similar to that of the bulk so that the dimensionality

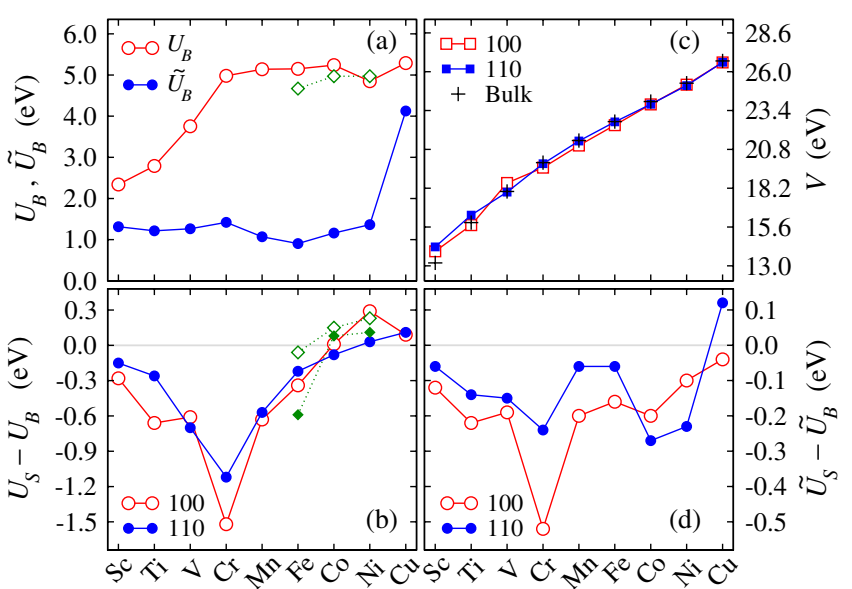

FIG. 2 (color online). (a) Partially screened $\left(U_{B}\right)$ and fully screened $\left(\tilde{U}_{B}\right)$ Coulomb interaction for the bulk $3 d$ TM series in the nonmagnetic state. With open diamonds we show the $U$ for ferromagnetic $\mathrm{Fe}, \mathrm{Co}$, and $\mathrm{Ni}$. (b) The difference between the surface and bulk $U$ values. Open (filled) diamonds show $U_{S}-U_{B}$ for the (100) [(110)] surface of ferromagnetic Fe, Co, and Ni. (c) Bare Coulomb interaction $V$ for bulk and surfaces. (d) The same as (b) for the fully screened Coulomb interaction $\tilde{U}$.

effect (i) wins over the electron-structure effect (ii), giving rise to enhanced Hubbard $U$ parameters.

For most other $3 d$ metals, the Hubbard $U$ parameter is reduced at the surface, too, but less than in the case of bcc $\mathrm{Cr}$, and for the late TMs the surface $U$ exceeds that of the bulk $U$. In Fig. 2(a) we present the partially screened $U_{B}$ and fully screened $\tilde{U}_{B}$ values for the bulk phases of the $3 d$ TMs in the nonmagnetic state. Results for the ferromagnetic ground states of $\mathrm{Fe}, \mathrm{Co}$, and $\mathrm{Ni}$ are also included for comparison. The difference between the surface and bulk $U$ and $\tilde{U}$ values, i.e., $U_{S}-U_{B}$ and $\tilde{U}_{S}-\tilde{U}_{B}$, is presented in Figs. 2(b) and 2(d) for the (100) and (110) surface. As seen, from $\mathrm{Sc}$ to Fe the Hubbard $U_{S}$ is reduced at both surfaces $\left(U_{S}-U_{B}<0\right)$, and Co is at the border, in which the Hubbard $U$ assumes similar values in the bulk and at the surface. Only at the $\mathrm{Ni}$ and $\mathrm{Cu}$ surface the $U$ is slightly larger. As for the difference of the fully screened Coulomb interaction, i.e., $\tilde{U}_{S}-\tilde{U}_{B}$, we obtain a qualitatively similar behavior, but the relative reduction of $\tilde{U}$ with respect to the bulk value is significantly larger. This is attributed to the fact that, in contrast to the Hubbard $U$, screening effects stemming from $3 d \rightarrow 3 d$ transitions contribute to the effective interaction, too. At the surface these virtual transitions take place within the surface states, which leads to very effective screening effects that give rise to the observed reduction of the $\tilde{U}$. It is important to point out that the variations of $U$ seen in Fig. 2 are not caused by different spreads of the Wannier function across the series, as can be seen from the bare Coulomb interaction $V$, which is presented in Fig. 2(c). As seen, bulk and surface $V$ values monotonically increase from $\mathrm{Sc}$ to $\mathrm{Cu}$, with no apparent 
difference between bulk and surface values. We note that the Hubbard $U$ values depend only little on the bare Coulomb interaction $V$ because in metals we are in the strong coupling limit, i.e., $v\left|P_{l}\right| \gg 1$, and thus $U \simeq-P_{l}^{-1}$. As for the surface $U_{S}$ of the ferromagnets $\mathrm{Fe}, \mathrm{Co}$, and $\mathrm{Ni}$, the same discussion holds. So far, we have not taken into account the surface relaxation, which is usually small $(<5 \%)$ for most of the $3 d$ TMs [28,29]. Only the bcc $V$ (100) surface possesses a sizeable inward relaxation $(\sim 11 \%)$, which results in a small change $(\sim 5 \%)$ of the calculated surface $U$ and $\tilde{U}$ values.

In contrast to metals, the Hubbard $U$ at insulator surfaces is much more strongly affected by the presence of surface states than the band narrowing. For example, the $\mathrm{NaCl}$ (100) surface does not exhibit any surface states [30], and the $U$ is enhanced. The slight changes in the electronic structure of the (110) surface (the gap is smaller, see Fig. 1) reduces the surface $U$ only very little so that it is still larger than the bulk value. The situation is similar for the $\mathrm{SrTiO}_{3}$ surface, for which the results are presented in Table I. In $\mathrm{MgO}$, on the other hand, both (100) and (110) surfaces exhibit surface states, which effectively reduce the band gap. For the (100) surface this gap reduction is around $0.6 \mathrm{eV}$ [31], while for the (110) surface we obtain a much stronger reduction, the surface states lie $3.3 \mathrm{eV}$ below the conduction-band minimum, i.e., close to the middle of the bulk GGA band gap, which amounts to $4.97 \mathrm{eV}$. The presence of the surface state strongly affects the screening properties with the consequence that the $U$ parameter is considerably reduced at the (110) surface, while it remains slightly above the bulk value in the case of the (100) surface. Analogously, localized states in the gap of an insulator stemming from defects such as vacancies, interstitial or substitutional impurities at surfaces lead also to a reduction of the surface $U$, but it is expected to be local, i.e., only the Hubbard $U$ parameters of the defect atom and nearby atoms are affected.

Finally we discuss the frequency dependence of the surface $U(\omega)$ considering the nonmagnetic state of bcc $\mathrm{Cr}$ and fcc Ni. The obtained results are presented in Fig. 3 and compared with the bulk calculations. We see that, apart from the substantial reduction of the static $U$ at the $\mathrm{Cr}$ surface, its frequency dependence is quite different from that of the bulk: $U_{S}(\omega)$ increases monotonically with frequency, and at about $2 \mathrm{eV}$ it crosses the $U_{B}(\omega)$ curve towards larger values, while $U_{B}(\omega)$ stays almost constant between 0 and $4 \mathrm{eV}$. For frequencies up to the plasmon frequency, $U_{S}(\omega)$ is mostly larger than $U_{B}(\omega)$. This behavior is not surprising because in the TMs the $3 d$ states close to the Fermi level are much more affected by the presence of the surface than the states at higher energies. Hence, with increasing frequency the polarization function becomes less sensitive to subtleties of the surface electronic structure, and at high frequencies the surface $U(\omega)$ tends to be larger than the bulk value due to the dimensionality effect.

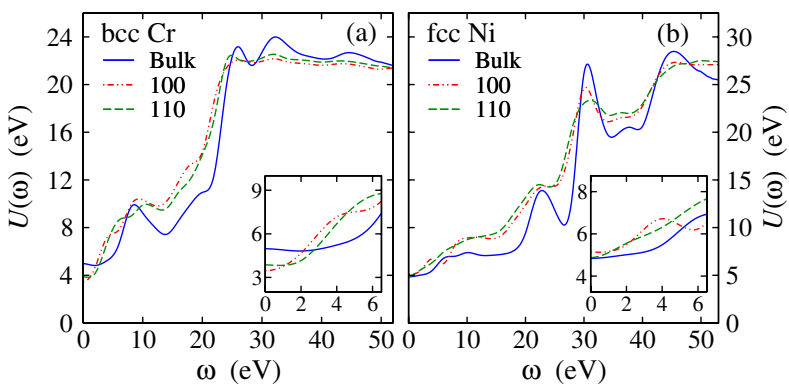

FIG. 3 (color online). (a) Frequency dependence of the bulk and surface Hubbard $U$ parameter for bcc Cr. In the inset we expand the low frequency region. (b) The same for fcc Ni.

In conclusion, by employing first-principles calculations in conjunction with the constrained random-phase approximation we have determined the strength of the Hubbard $U$ parameter at metal and insulator surfaces. We showed that $U$ does not always increase at the surface as commonly expected. In fact, it decreases at most of the TM surfaces as well as insulator surfaces with pronounced surface states. We found that surface states and the effective band narrowing play an important role for the strength of the Hubbard $U$ at surfaces. The fact that the $U$ parameter can be made to increase as well as decrease at surfaces offers new possibilities in designing materials with tunable correlations.

Fruitful discussions with A. Liebsch, G. Bihlmayer, D. Wortmann, A. Schindlmayr, and A. Lichtenstein are gratefully acknowledged. This work has been supported by the DFG through the Research Unit FOR-1346.

*e.sasioglu@fz-juelich.de

[1] J. Zaanen and G. A. Sawatzky, J. Solid State Chem. 88, 8 (1990).

[2] S.-K. Mo, J. D. Denlinger, H.-D. Kim, J.-H. Park, J. W. Allen, A. Sekiyama, A. Yamasaki, K. Kadono, S. Suga, Y. Saitoh, T. Muro, P. Metcalf, G. Keller, K. Held, V. Eyert, V. I. Anisimov, and D. Vollhardt, Phys. Rev. Lett. 90, 186403 (2003).

[3] A. Goldoni, A. Baraldi, G. Comelli, S. Lizzit, and G. Paolucci, Phys. Rev. Lett. 82, 3156 (1999).

[4] N. Kamakura, Y. Takata, T. Tokushima, Y. Harada, A. Chainani, K. Kobayashi, and S. Shin, Europhys. Lett. 67, 240 (2004).

[5] M. Potthoff and W. Nolting, Phys. Rev. B 59, 2549 (1999).

[6] A. Liebsch, Phys. Rev. Lett. 90, 096401 (2003).

[7] A. Grechnev, I. Di Marco, M. I. Katsnelson, A. I. Lichtenstein, J. Wills, and O. Eriksson, Phys. Rev. B 76, 035107 (2007).

[8] T. Kotani, J. Phys. Condens. Matter 12, 2413 (2000).

[9] I. Schnell, G. Czycholl, and R. C. Albers, Phys. Rev. B 65, 075103 (2002); I. V. Solovyev and M. Imada, Phys. Rev. B 71, 045103 (2005).

[10] P.H. Dederichs, S. Blügel, R. Zeller, and H. Akai, Phys. Rev. Lett. 53, 2512 (1984); V.I. Anisimov and O. Gunnarsson, Phys. Rev. B 43, 7570 (1991); 
M. Cococcioni and S. de Gironcoli, Phys. Rev. B 71, 035105 (2005); K. Nakamura, R. Arita, Y. Yoshimoto, and S. Tsuneyuki, Phys. Rev. B 74, 235113 (2006).

[11] F. Aryasetiawan, M. Imada, A. Georges, G. Kotliar, S. Biermann, and A. I. Lichtenstein, Phys. Rev. B 70, 195104 (2004); F. Aryasetiawan, K. Karlsson, O. Jepsen, and U. Schönberger, Phys. Rev. B 74, 125106 (2006); T. Miyake, F. Aryasetiawan, and M. Imada, Phys. Rev. B 80, 155134 (2009).

[12] E. Şaşığlu, C. Friedrich, and S. Blügel, Phys. Rev. B 83, 121101(R) (2011).

[13] P. Werner, M. Casula, T. Miyake, F. Aryasetiawan, A. J. Millis, and S. Biermann, Nature Phys. 8, 331 (2012).

[14] T. O. Wehling, E. Şaşığlu, C. Friedrich, A.I. Lichtenstein, M. I. Katsnelson, and S. Blügel, Phys. Rev. Lett. 106, 236805 (2011).

[15] I. V. Solovyev, Phys. Rev. Lett. 95, 267205 (2005).

[16] T. Ando, A. B. Fowler, and F. Stern, Rev. Mod. Phys. 54, 437 (1982).

[17] L. Reining and R. Del Sole, Phys. Rev. B 38, 12768 (1988).

[18] N. Marzari and D. Vanderbilt, Phys. Rev. B 56, 12847 (1997).

[19] http://www.flapw.de.

[20] J. P. Perdew, K. Burke, and M. Ernzerhof, Phys. Rev. Lett. 77, 3865 (1996).
[21] A. A. Mostofi, J.R. Yates, Y.-S. Lee, I. Souza, D. Vanderbilt, and N. Marzari, Comput. Phys. Commun. 178, 685 (2008).

[22] F. Freimuth, Y. Mokrousov, D. Wortmann, S. Heinze, and S. Blügel, Phys. Rev. B 78, 035120 (2008).

[23] C. Friedrich, S. Blügel, and A. Schindlmayr, Phys. Rev. B 81, 125102 (2010).

[24] E. Şaşığlu, A. Schindlmayr, C. Friedrich, F. Freimuth, and S. Blügel, Phys. Rev. B 81, 054434 (2010).

[25] K. Karlsson, F. Aryasetiawan, and O. Jepsen, Phys. Rev. B 81, 245113 (2010).

[26] B-C. Shih, Y. Zhang, W. Zhang, and P. Zhang, Phys. Rev. B 85, 045132 (2012).

[27] O. Yu. Kolesnychenko, G. M. M. Heijnen, A. K. Zhuravlev, R. de Kort, M. I. Katsnelson, A.I. Lichtenstein, and H. van Kempen, Phys. Rev. B 72, 085456 (2005).

[28] J. L. F. DaSilva, C. Stampfl, and M. Scheffler, Surf. Sci. 600, 703 (2006).

[29] G. Bihlmayer, T. Asada, and S. Blügel, Phys. Rev. B 62, R11937 (2000).

[30] B. Li, A. Michaelides, and M. Scheffler, Phys. Rev. B 76, 075401 (2007).

[31] U. Schönberger and F. Aryasetiawan, Phys. Rev. B 52, 8788 (1995). 